\title{
Motor Task Processing After Constraint- Induced Movement Therapy in Children With Cerebral Palsy: A Case Series
}

\author{
Karen P.Y. Liu' ${ }^{1,2 *}$, Michael C.C. Kuo ${ }^{(\circledR)}$, Kin-Hung Ting ${ }^{(®)}$ \\ ${ }^{1}$ School of Health Sciences, Western Sydney University, Australia \\ ${ }^{2}$ Translational Health Research Institute, Western Sydney University, Australia \\ ${ }^{3}$ Department of Rehabilitation and Social Sciences, Tung Wah College, Hong Kong \\ ${ }^{4}$ University Research Facility in Behavioral and Systems Neuroscience, The Hong Kong Polytechnic University, Hong Kong
}

\begin{abstract}
Constraint-induced movement therapy (CIMT) has shown positive results in children with hemiplegic cerebral palsy (CP). However, studies on neural basis of such functional gains are limited. This study reports the event-related potential (ERP) changes in two children with hemiplegic CP after receiving CIMT for three weeks. Both cases were nine years old, had a diagnosis of left hemiplegic CP, had normal intelligence, and were able to extend the wrist at least $20^{\circ}$ and the metacarpophalangeal joint at least $10^{\circ}$ from full flexion. Before and after the three-week intervention, the children participated in ERP sessions with a choice reaction task to capture the changes in neural mechanism after intervention. Both children exhibited improvement in reaction time (RT) in both hand tasks after the intervention. The improvement was larger in the affected hand than the unaffected hand. Improved accuracy rate (AC) and shortened P300 latencies in the affected hand were also demonstrated in both cases. Topographical maps showed that in centro-parietal regions, patterns shifted from central and left-lateralized to more central and right-lateralized. CMIT was a useful method in improving upper limb function in our cases.

Keywords: Constraint-induced movement therapy; Cerebral palsy; Upper limb functional regain; Event-related potentials.
\end{abstract}

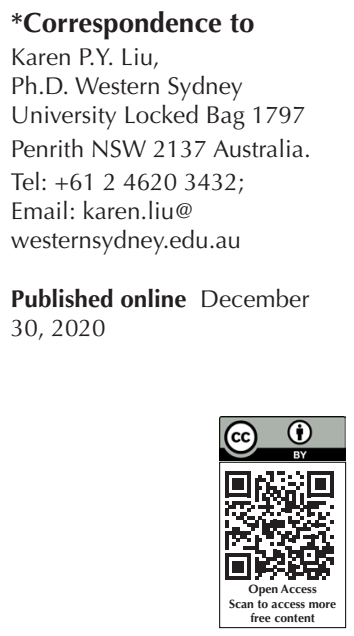

*Correspondence to

University Locked Bag 1797

Penrith NSW 2137 Australia.

Tel: +6124620 3432;

Email: karen.liu@

Published online December 30, 2020

Citation: Liu KPY, Kuo MCC, Ting KH. Motor task processing after constraint-induced movement therapy in children with cerebral palsy: a case series. Clin Neurosci J. 2021;8(1):44-47. doi:10.34172/icnj.2021.09.

\section{Introduction}

Constraint-induced movement therapy (CIMT) was initially developed for people with stroke. ${ }^{1-3}$ It has also been used for children with hemiplegic cerebral palsy (CP) with promising results. ${ }^{4,5}$ CIMT involves restraining the unaffected limb so that participants would be forced to use the affected limb. ${ }^{6}$ This procedure was theorized to overcome learned non-use or developmental disregard and induces a use-dependent plastic brain reorganization.

Evidence of cortical reorganization from functional magnetic resonance imaging (fMRI) studies include increased cortical representation of the affected extremity, ${ }^{7}$ increased activations in the primary sensorimotor cortex of the hemisphere with lesions, ${ }^{8}$ and increased bilateral cortical activation in the sensorimotor cortex and a shift in laterality from the ipsilateral hemisphere to the contralateral hemisphere. ${ }^{9}$ To our knowledge, there have been no reports on event-related potentials (ERPs) with respect to the use of CIMT on children with CP. Considering their temporal characteristics in identifying neural activities, ERPs allow more precise investigation of cognitive processes associated with CIMT. ${ }^{10}$

This study reports the change in reaction time (RT) and accuracy rate $(\mathrm{AC})$ and the ERP changes of a reaction task in two children with hemiplegic CP after receiving CIMT. Specifically, we investigated the P300 component, which indexes stimulus evaluation and processing, ${ }^{11}$ as well as the topographical changes.

\section{Materials and Methods \\ Participants}

The parents of the participants and the participants themselves both gave their written consent to report the results. Children with hemiplegic $\mathrm{CP}$ with no previous experience of CIMT were recruited. The two participants (one boy and one girl) were nine years old, both had left hemiplegic CP with normal intelligence, and were able to extend the wrist at least $20^{\circ}$ and the metacarpophalangeal joint at least $10^{\circ}$ from full flexion. They had no prior experience of CIMT.

(C) 2021 The Author(s). This is an open access article distributed under the terms of the Creative Commons Attribution License (http:// creativecommons.org/licenses/by/4.0/), which permits unrestricted use, distribution, and reproduction in any medium, provided the original work is properly cited. 


\section{CIMT and Procedure}

Both children received CIMT for three weeks, five days a week, and six hours a day. They wore a comfortable cotton sling on the unaffected arm. During each 6-hour restraint, a one-hour structured practice incorporating the principle of shaping and repetitive practice was done at the occupational therapy clinic. ${ }^{12}$ Prior to the initiation of the treatment and after a three-week period, the children participated in the ERP sessions of a choice reaction task.

\section{Choice Reaction Task and ERP Recording}

Each child was required to complete a choice reaction task (a two-choice task) separately for the affected and unaffected hands. They responded by pressing the buttons on an oversized number pad with keys that could be pressed with the index finger or the thumb. Stimuli were pictures of an outlined hand with the responding digit highlighted. When the child saw a thumb highlighted, he or she was required to press the thumb key with his/ her thumb. Alternatively, he or she would press the index finger key with the index finger if the index finger was highlighted in the picture. These pictures were modified in a way that the required fingers could be seen easily. Before the experiment, the children were trained to get familiarized with the task. They also completed a practice block of 20 trials to assure that they understood the instruction and the task. The allocation of time in each trial is shown in Figure 1.

There was a total of 8 blocks. Each block was composed of 40 trials. The presentation of the highlighted thumb or index fingers (50\% probability each) was randomized in each block.

ERPswere recorded from a 64-channel array of electrodes placed in an extended 10-20 system. The ground electrode was placed at the forehead and these channels were leftmastoid referenced and were re-referenced offline to a linked-mastoid reference. The SymAmps2 EEG amplifier (Compumedics NeuroScan, Charlotte, NC) were used for

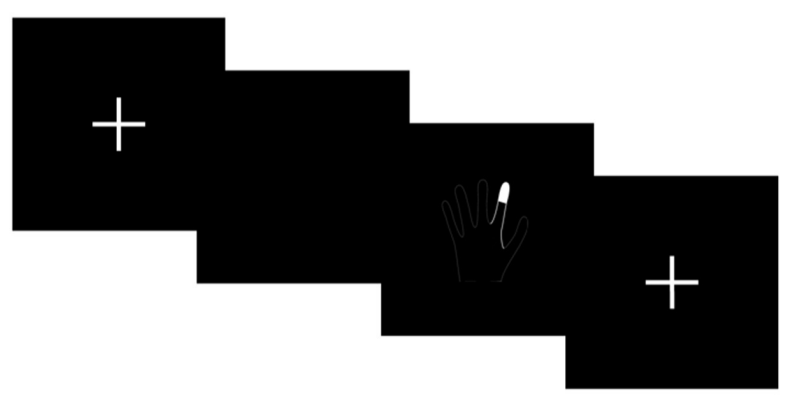

$\begin{array}{cccc}+ & \text { Blackout } & \text { Picture } 300 \mathrm{~ms} & \text { Repeat } \\ 1000 \mathrm{~ms} & 500-1200 \mathrm{~ms} & \text { Response } 2500 \mathrm{~ms} & \end{array}$

Figure 1. A Schematic Display of a Trial (With Left Index Finger Highlighted). data capturing. The band-pass filter was set at $0.5-40 \mathrm{~Hz}$, and sampling at $1 \mathrm{kHz}$. Impedances were set at $<5 \mathrm{k} \Omega$. Vertical and horizontal electrooculograms were recorded to monitor eye movements.

\section{Data Analysis}

Data management and analysis of the ERPs were performed with the Scan Version 4.5 (Compumedics NeuroScan, Charlotte, NC). RT and AC of the children's responses were captured as behavioral measures. Recordings were first merged with behavioral data and then linked to an averaged mastoid reference. After that, ocular rejection was applied on all channels. This was followed by filtering (zero phase shift band pass filter 0.1-20 Hz). Stimulus-locked epochs were cut from -200 $\mathrm{ms}$ to $800 \mathrm{~ms}$ for the male participant and $-200 \mathrm{~ms}$ to $1150 \mathrm{~ms}$ for the female participant and the baseline was corrected to the pre-stimulus interval. Incorrect trials and trials containing artifacts $( \pm 75 \mathrm{uV})$ were rejected. The remaining trials were then averaged for analysis. Peak latency for P300 was measured between $250 \mathrm{~ms}$ and 700 ms from the channel $\mathrm{CPz}$ and $\mathrm{Pz}$.

\section{Results}

\section{Behavioral Results}

Both children exhibited improvements in RT in both tasks for the right and left hands after the three-week intervention. The improvement was larger in the affected left hand $(733 \mathrm{~ms}$ to $659 \mathrm{~ms}$ for the boy and $1104 \mathrm{~ms}$ to $1051 \mathrm{~ms}$ for the girl) than the unaffected right hand (583 $\mathrm{ms}$ to $573 \mathrm{~ms}$ for the boy and $894 \mathrm{~ms}$ to $882 \mathrm{~ms}$ for the girl). Improved $\mathrm{AC}$ in the affected hand was also demonstrated in both cases after the treatment (79\%-89\% for the boy and $89 \%-94 \%$ for the girl).

\section{ERP Results}

P300 latency is shown in Table 1. P300 latency was shorter post CIMT in both cases. Figure 2 presents the ERP waveforms of a channel $\mathrm{CPz}$.

Figure 3 show the topographic map of the children before and after CIMT. In both cases, in the centroparietal regions, patterns shifted from central and leftlateralized to more central and right-lateralized (from 300-500 ms for the boy and from 420-840 ms for the girl after stimulus presentation).

\section{Discussion}

The goal of the present study was to investigate P300 as

Table 1. P300 Latency for the Two Clinical Cases

\begin{tabular}{lcc}
\hline & \multicolumn{2}{c}{ P300 Latency (ms) } \\
\cline { 2 - 3 } & Before CIMT & After CIMT \\
\hline Boy & 332 & 274 \\
Girl & 626 & 417 \\
\hline
\end{tabular}


(a)

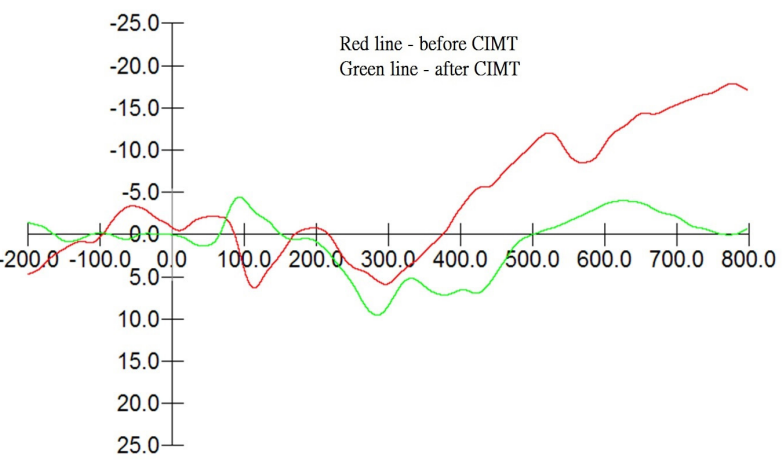

ms

(b)

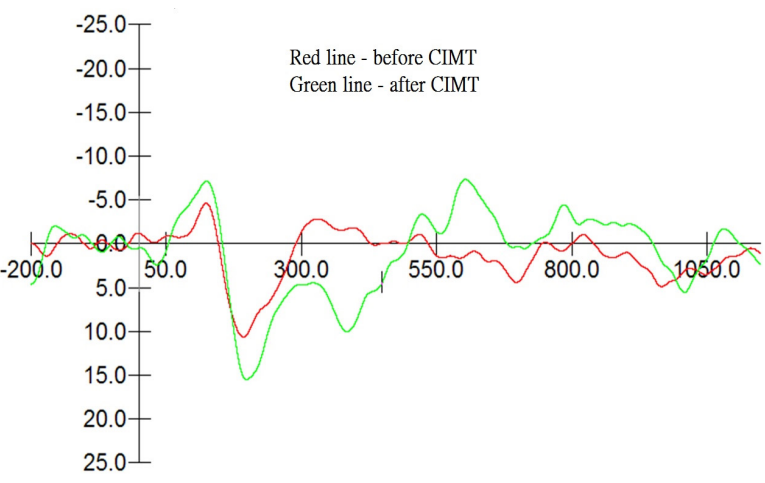

$\mathrm{ms}$

Figure 2. (a) ERPs at CPz of the Boy. (b) ERPs at CPz of the Girl.

(a)
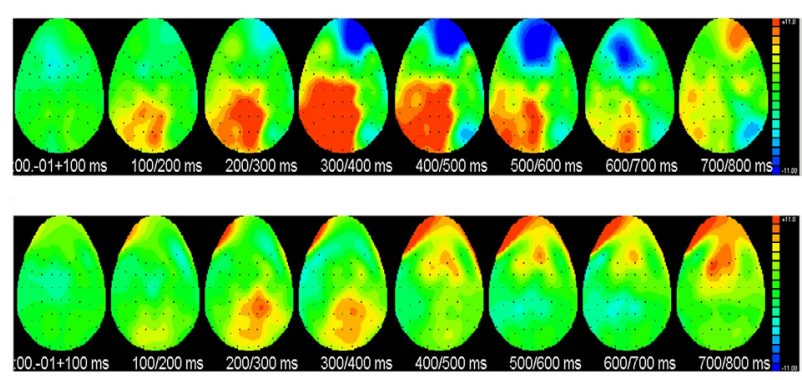

(b)
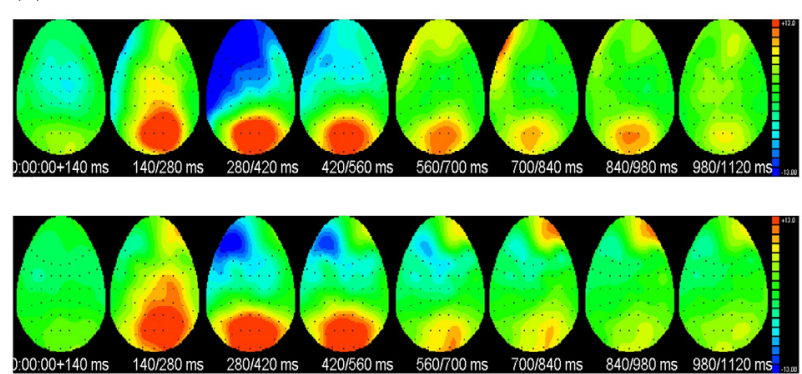

Figure 3. (a) Topographical Maps of the Boy Before and After CIMT. (b) Topographical Maps of the Girl Before and After CIMT. Note. Upper row represents pre- and lower row post-treatment. Each map is separated by $100 \mathrm{~ms}$. well as topographical changes after the children received CIMT. Behavioral data showed shorter RT and higher AC after treatment. P300 latency was shortened as well. Possible meaningful topographical change was evident in the centro-parietal regions.

The RT improvement was larger in the affected left hand than the unaffected right hand in both children. RT improvement in the unaffected hand may be attributed to the learning effect since both children had a second opportunity to perform exactly the same task. The larger RT changes in the affected hand may possibly be accounted for by the treatment effect of CIMT.

Previous findings have suggested that there may be a delay in P300 latency in similar motor tasks for people with brain injuries as compared with healthy participants. ${ }^{13,14}$ P300 could be used as a marker for attentional processing as well as stimulus evaluation processes. ${ }^{11,14}$ While there was no normal group for comparison, the latency of P300 component was shorter after CIMT for these two children. Thus, it may indicate that changes in the premotor stage of the cognitive processes related to this choice reaction task could have occurred because of the treatment provided. This was an important additional finding. Other than the cortical reorganization as revealed by fMRI, in these two cases, CIMT enhanced very initial attentional processing and stimulus evaluation that are important in accurate motor performance. ${ }^{15}$ This offers a sound explanation of the clinical evidence that CIMT improved motor functions of people with hemiplegia.

Some evidence of motor-related changes may be gathered from the topographical maps of the two children. In this regard, the activities in the posterior aspect of the brain appear to shift rightward from a more central or left-lateralized pattern. As shown in other fMRI studies, cortical reorganization post-CIMT may include increased activations in the primary sensorimotor cortex of the lesioned hemisphere ${ }^{7,8}$ and a shift in laterality from the ipsilateral hemisphere to the contralateral hemisphere. ${ }^{9}$ Therefore, the topographical results in this study could also be interpreted along similar lines assuming that the neural substrates generating these topographical patterns are similar.

\section{Conclusion}

The results of this case study showed that CIMT could implicate both processes related to interpreting incoming stimulus (attention, perception, and evaluation) as well as motor processes for response execution. The findings of this study may provide additional information that further strengthen the clinical evidence of CIMT. More participants will be needed to confirm these findings and permit statistical analysis.

\section{Conflict of Interest}

The authors declare that they have no conflict of interests. 


\section{Acknowledgements}

The authors would like to thank the children for the participation and their occupational therapists for their assistance during the data collection period.

\section{Authors' Contributions}

$\mathrm{KL}, \mathrm{KT}$ and $\mathrm{MK}$ were involved in the study design and the data collection process. $\mathrm{KL}$ and MK drafted the manuscript, $\mathrm{KL}, \mathrm{KT}$ and MK finalized the manuscript. All authors are accountable for all sections of the manuscript and declare that it is written originally and there is no data fabrication and falsification.

\section{Funding/Support}

None.

\section{Ethical Statement}

The procedures followed were in accordance with the ethical standards of the responsible committee on human experimentation and with the Helsinki Declaration of 1975, as revised in 1983. The study was approved by the Hong Kong Polytechnic University, Department of Rehabilitation Sciences research ethics committee. The participants were provided with an information sheet and the study was explained to them verbally, providing an opportunity for them to discuss any concerns prior to providing written consent. Informed written consent was obtained for the participants before the start of the study. Confidentiality of information was maintained throughout the study.

\section{Availability of Data and Materials}

The datasets used and/or analyzed during the current study are available from the Corresponding author on reasonable request.

\section{References}

1. Taub E, Morris DM. Constraint-induced movement therapy to enhance recovery after stroke. Curr Atheroscler Rep. 2001;3(4):279-86. doi: 10.1007/s11883-001-0020-0.

2. Lang KC, Thompson PA, Wolf SL. The EXCITE Trial: reacquiring upper-extremity task performance with early versus late delivery of constraint therapy. Neurorehabil Neural Repair. 2013;27(7):654-63. doi: 10.1177/1545968313481281.

3. Wu CY, Chen $\mathrm{CL}$, Tang SF, Lin $\mathrm{KC}$, Huang YY. Kinematic and clinical analyses of upper-extremity movements after constraint-induced movement therapy in patients with stroke: a randomized controlled trial. Arch Phys Med Rehabil. 2007;88(8):964-70. doi: 10.1016/j.apmr.2007.05.012.

4. Senesac C, Helsel PV, Breton J. Modified constraint induced movement therapy for child with hemiplegia. Pediatr Phys Ther. 2006;18(1):104.

5. Gordon AM, Charles J, Wolf SL. Efficacy of constraint-induced movement therapy on involved upper-extremity use in children with hemiplegic cerebral palsy is not age-dependent. Pediatrics. 2006;117(3):e363-73. doi: 10.1542/peds.20051009.

6. Bowman MH, Mark VW, Taub E. Constraint-induced movement therapy for restoration of upper-limb function: introduction. In: Söderback I, ed. International Handbook of Occupational Therapy Interventions. Cham: Springer; 2015. p. 573-85. doi: 10.1007/978-3-319-08141-0_40.

7. Szaflarski JP, Page SJ, Kissela BM, Lee JH, Levine P, Strakowski SM. Cortical reorganization following modified constraintinduced movement therapy: a study of 4 patients with chronic stroke. Arch Phys Med Rehabil. 2006;87(8):1052-8. doi: 10.1016/j.apmr.2006.04.018.

8. Jünger $\mathrm{CH}$, Linder $\mathrm{M}$, Walther $\mathrm{M}$, Berweck $\mathrm{S}$, Mall V, Staudt M. Cortical neuromodulation by constraint induced therapy in congenital hemiparesis-an fMRI study. Clin Neurophysiol. 2007;118(4):e51-e2. doi: 10.1016/j.clinph.2006.11.127.

9. Sutcliffe TL, Gaetz WC, Logan WJ, Cheyne DO, Fehlings DL. Cortical reorganization after modified constraintinduced movement therapy in pediatric hemiplegic cerebral palsy. J Child Neurol. 2007;22(11):1281-7. doi: 10.1177/0883073807307084.

10. Maitre NL, Henderson G, Gogliotti S, Pearson J, Simmons A, Wang L, et al. Feasibility of event-related potential methodology to evaluate changes in cortical processing after rehabilitation in children with cerebral palsy: a pilot study. J Clin Exp Neuropsychol. 2014;36(7):669-79. doi: 10.1080/13803395.2014.925094.

11. van Dinteren R, Arns M, Jongsma ML, Kessels RP. P300 development across the lifespan: a systematic review and meta-analysis. PLoS One. 2014;9(2):e87347. doi: 10.1371/ journal.pone.0087347.

12. Abd El-Kafy EM, Elshemy SA, Alghamdi MS. Effect of constraint-induced therapy on upper limb functions: a randomized control trial. Scand J Occup Ther. 2014;21(1):1123. doi: 10.3109/11038128.2013.837505.

13. Hirata K, Katayama S, Yamazaki K, Fujikane M, Katayama K. Electric field distribution of event-related potentials in stroke patients. Brain Topogr. 1996;8(3):279-84. doi: 10.1007/ bf01184785.

14. Lew HL, Gray M, Poole JH. Simultaneous measurement of perceptual and motor cortical potentials: implications for assessing information processing in traumatic brain injury. Am J Phys Med Rehabil. 2009;88(1):1-6. doi: 10.1097/ PHM.0b013e3181911102.

15. Chung JW, Ofori E, Misra G, Hess CW, Vaillancourt DE. Beta-band activity and connectivity in sensorimotor and parietal cortex are important for accurate motor performance. Neuroimage. 2017;144(Pt A):164-73. doi: 10.1016/j. neuroimage.2016.10.008. 